
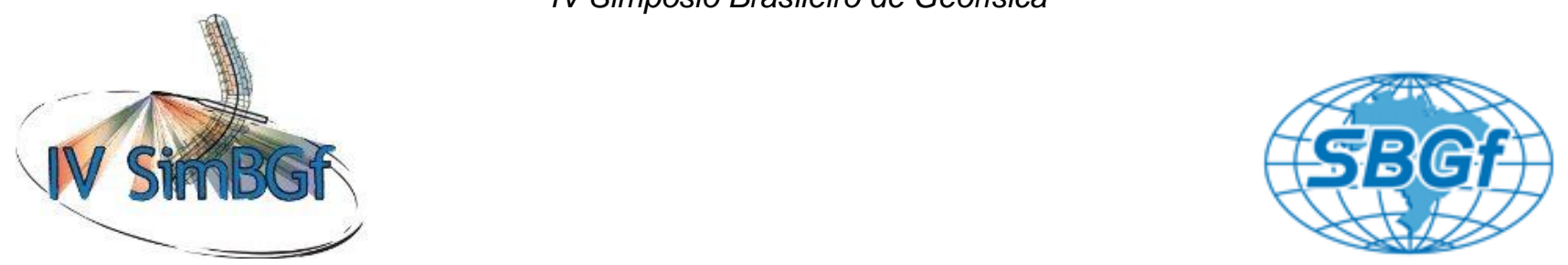

\title{
Associação espacial preliminar entre produtividade de poços de abastecimento e variáveis geológicas e geofísicas no Sudeoste do Paraná
}

Oderson Antônio de Souza Filho (NAC/CPRM), Alessandra de Barros e Silva Bongiolo (LPGA/UFPR), Gustavo Barbosa Athayde (SANEPAR, LHP/UFPR), Francisco José Fonseca Ferreira (LPGA/UFPR), Luís Gustavo de Castro (LPGA/UFPR)

Copyright 2010, SBGf - Sociedade Brasileira de Geofísica

Este texto foi preparado para a apresentação no IV Simpósio Brasileiro de Geofísica, Brasília, 14 a 17 de novembro de 2010. Seu conteúdo foi revisado pelo Comitê Técnico do IV SimBGf, mas não necessariamente representa a opinião da SBGf ou de seus associados. É proibida a reprodução total ou parcial deste material para propósitos comerciais sem prévia autorização da SBGf.

\section{Resumo}

Com o objetivo de subsidiar a Carta de Água Subterrânea do Estado do Paraná, foi escolhida uma área-piloto do sudoeste do estado para testar metodologias que identifiquem uma possível associação espacial entre a produtividade de poços e condicionantes geológicos, morfológicos, estruturais e geofísicos em vulcanitos do Grupo Serra Geral, na Bacia do Paraná. Como resultado preliminar, maiores vazões estão associadas a orientações NW-SE a N-S de lineamentos estruturais e orientações N-S a NE-SW de gradientes magnéticos que cortam basaltos microfaneríticos e, declividades acentuadas do terreno não favorecem a produtividade de poços.

\section{Introdução}

O Serviço Geológico do Brasil/CPRM tem como uma das diretrizes principais, a geração de informação geológica e hidrogeológica básica para o país. Nesse contexto, o presente trabalho está inserido no programa de execução das cartas geológicas e hidrogeológicas estaduais. Para a elaboração destas cartas no estado, o Laboratório de Pesquisas em Geofísica Aplicada/LPGA da UFPR, participa com a geração de produtos geofísicos de suporte ao mapeamento geológico e hidrogeológico. A área-piloto (Figura 1) localiza-se na porção sudoeste do estado do Paraná, junta das fronteiras com a Argentina e - Paraguai. A morfologia é suavemente ondulada a plana, com altitudes variando entes 120 e 700 metros com escarpas e declives maiores próximos ás drenagens principais. O clima da região é subtropical, com geadas pouco freqüentes e tendência de concentração das chuvas no verão e na primavera.

A geologia compreende as rochas vulcânicas cretácias do Grupo Serra Geral, que, segundo Schobbenhaus et al. (2003) subdividem-se nas formações: Campo Êre (basalto tholeiítico (MORB), com textura microfanerítica; Cordilheira alta (basalto tholeiítico (MORB), com textura microfanerítica); Campos Novos (basalto com textura intergranular); Nova Laranjeiras (basalto com textura vítrea); Capanema (basalto com textura microfanerítica); Serra Geral: (basalto e dacito). concomitante aos basaltos, ocorrem os arenitos quartzosos e bem selecionados da Formação Rio Paraná, também do
Cretácio. Sobrepostos a estes, estão os depósitos aluvionares do Quaternário.

Dentre as principais unidades aqüíferas do Estado do Paraná (Figura 2), o Aqüífero Serra Geral (ASG) destacase em função de sua área de abrangência, importância para o abastecimento publico e qualidade das águas in natura.

A importância estratégica do ASG é proporcional à dificuldade exploratória (poços com vazões representativas). Tal dificuldade dá-se em função, principalmente, da anisotropia estrutural do aqüífero.

A maior freqüência das entradas de água nos poços perfurados na Fm. Serra Geral está localizada até os 110 $\mathrm{m}$ de profundidade, nessa faixa as vazões variam de 1 a $70 \mathrm{~m}^{3} / \mathrm{h}$ (ROSA FILHO et al., 1987).

Uma abordagem regional realizada por Rosa Filho e Hindi (2006) descreve para o ASG, no Estado do Paraná, teores de sólidos totais dissolvidos entre 100 e $150 \mathrm{mg} / \mathrm{L}$, $\mathrm{pH}$ entre 6,6 a 7,2 e dureza em torno de $40 \mathrm{mg}-\mathrm{CaCO}_{3} / \mathrm{L}$. $\mathrm{O}$ teor médio de cálcio é $9 \mathrm{mg} \cdot \mathrm{L}^{-1}$. As concentrações de magnésio variam de 3,5 a $6,5 \mathrm{mg} \cdot \mathrm{L}^{-1}$, as de sódio, entre 1,2 a $3,7 \mathrm{mg} \cdot \mathrm{L}^{-1}$, e as de potássio, entre 1,5 a $3 \mathrm{mg} \cdot \mathrm{L}^{-1}$. O teor médio de bicarbonato é $38 \mathrm{mg} \cdot \mathrm{L}^{-1}$, o de cloreto, 1,5 $\mathrm{mg} \cdot \mathrm{L}^{-1}$ e o de sulfato, $2,5 \mathrm{mg} \cdot \mathrm{L}^{-1}$.

\section{Metodologia/ Problema Investigado}

A geologia foi extraída da Carta Geológica ao Milionésimo, Folha SG.22 (Schobbenhaus et al., 2003), além dos dados magnetométricos do levantamento aerogeofísico Borda Oeste da Bacia do Paraná (ENCAL, 1989) e dados de poços da Companhia de Saneamento do Paraná/SANEPAR. O levantamento aerogeofísico foi executado a altura constante de $1 \mathrm{~km}$ do terreno, com linhas de aquisição N-S, espaçadas de $3 \mathrm{~km}$ e linhas de controle $\mathrm{E}-\mathrm{W}$, espaçadas de $18 \mathrm{~km}$, resultando numa medida a cada $1 \mathrm{~km}$ no terreno. $O$ sensor aeromagnetométrico, Geometrics, possui taxa de leitura de 1 segundo e sensibilidade de $1 \mathrm{nT}$.

Os dados magnetométricos de campo (Figuras 3a e 3b) total foram micronivelados e reduzidos do modelo geomagnético internacional/IGRF, constituindo os dados do campo magnético anômalo-CMA. Para eliminar ruídos de alta freqüência e caracterizar os gradientes magnéticos mais importantes, foi aplicado ao CMA o algoritmo de continuidade ascendente para $500 \mathrm{~m}$ e, a partir deste, foram testados vários métodos para realce de gradientes de anomalias orientadas e unidades geomagnéticas, dentre eles, gradiente horizontal totalGHT (Cordell and Grauch, 1985), amplitude do sinal 
analítico-ASA (Nabighian, 1972, 1974; Roest, et al. 1992), fase do sinal analítico-ISA (Miller e Singh, 1994) e gradiente vertical-GZ.

Os gradientes orientados relacionados a diques, falhas ou zonas de fraqueza, foram melhor realçados pela aplicação do algoritmo de inclinação do sinal analítico aos valores do gradiente horizontal total (ISA-GHT, Ferreira et al., neste simpósio). Domínios magnéticos foram discriminados em classes de intensidade magnética do CMA, auxiliados pela interpretação dos produtos de ASA, GZ e ISA-GHT.

A partir do modelo digital de terreno, foi gerado o mapa de declividade (graus). Este modelo é originário dos dados da missão SRTM (Rabus et al., 2003) e, cedido pela CPRM.

O banco de dados de poços contém 155 registros de vazão, que foram divididos para a aplicação na modelagem espacial: 100 poços foram usados na elaboração dos modelos (poços para treinamento); 55 poços servirão para teste dos modelos ao final do projeto (Tabela 1). Nos dois subconjuntos de poços (arquivos de pontos), foram calculadas áreas de proximidade de 300 $\mathrm{m}$ de raio para cada poço, que representam a área de influência hidrogeológica destes poços no terreno. O critério da área de influência foi definido como metade do tamanho do pixel dos grids magnetométricos.

Tabela 1 - Estatística dos conjuntos de poços de treinamento e de teste das análises espaciais.

\begin{tabular}{|l|l|l|l|l|l|}
\hline \multicolumn{2}{|l|}{$\begin{array}{l}\text { Número de Poços } \\
\text { (\% do total) }\end{array}$} & $\begin{array}{l}\mathrm{Q}_{\min } \\
\left(\mathrm{m}^{3} / \mathrm{h}\right)\end{array}$ & $\begin{array}{l}\mathrm{Q}_{\max } \\
\left(\mathrm{m}^{3} / \mathrm{h}\right)\end{array}$ & $\begin{array}{l}\text { Média } \\
\left(\mathrm{m}^{3} / \mathrm{h}\right)\end{array}$ & $\begin{array}{l}\text { Desvio } \\
\text { Padrão }\end{array}$ \\
\hline Total & $\begin{array}{l}155 \\
(100 \%)\end{array}$ & 3 & 250 & 42,81 & 41,73 \\
\hline Treinamento & $100(65 \%)$ & 3 & 250 & 42,60 & 43,60 \\
\hline Teste & $55(35 \%)$ & 3 & 165 & 43,18 & 38,08 \\
\hline
\end{tabular}

Para representar a produtividade dos poços, estes foram classificados pela vazão. Posteriormente, os dois conjuntos foram transformados em arquivos raster, definidos pelo atributo de classes de vazões, conforme a Tabela 2.

Tabela 2 - Classes de produtividades de poços de treinamento e de teste, segundo os intervalos de vazões e representatividade, dada pelo número de pixels.

\begin{tabular}{|l|l|l|l|}
\hline \multirow{2}{*}{$\begin{array}{c}\text { Classe de } \\
\text { Produtividade }\end{array}$} & $\begin{array}{c}\text { Intervalo } \\
\text { de Vazão } \\
\left(\mathrm{m}^{3} / \mathrm{h}\right)\end{array}$ & \multicolumn{2}{|c|}{$\begin{array}{c}\text { Representatividade de poços em } \\
\mathrm{n}^{\text {0. }} \text { de pixels de } 300 \mathrm{~m}\left(\mathrm{e} \mathrm{n}^{\circ} \mathrm{de}\right. \\
\text { poços })\end{array}$} \\
\cline { 3 - 5 } & & Treinamento (100) & Teste (55) \\
\hline Muito Baixa & $3-6$ & $178(9)$ & $76(4)$ \\
\hline Baixa & $7-15$ & $323(17)$ & $175(9)$ \\
\hline Média & $16-29$ & $476(28)$ & $212(11)$ \\
\hline Alta & $30-50$ & $357(22)$ & $318(17)$ \\
\hline Muito Alta & $54-250$ & $449(24)$ & $258(14)$ \\
\hline
\end{tabular}

Basaltos são aquíferos fraturados cuja potencialidade hídrica depende intrinsicamente de descontinuidade (estruturas e vesículas) e grau de intemperismo e, externamente, do clima, morfologia, zona de recarga, entre outros. Assim, diferentes conjuntos de dados podem revelar características geológicas, morfológicas e estruturais espacialmente associadas a vazões de poços, em outras palavras, indicadores preditivos para água subterrânea.

Para quantificar o quão característico (ou não) os dados geológicos, geofísicos, estruturais e morfológicos são de locais com poços de alta e baixa vazão foi aplicada a técnica de razão de probabilidades (Lee et al., 2001). Também chamada de pesos ( $W$ ), a técnica descreve a probabilidade de uma classe de um tema evidencial (litologia, dado magnético, declividade) estar associado com uma evidência de estudo (classe de vazão de poços de treinamento), através do cálculo da sobreposição de pixels de arquivos do tipo raster. A Equação 1 descreve a técnica citada, onde: $\mathrm{RP}=$ Razão de Probabilidade; Pixelsoverlap = área de sobreposição entre uma camada evidencial e uma classe de vazão; Pixels evid = área de uma classe de tema evidencial ocupa em relação a área de estudo; Pixels $_{\text {classe }_{\imath}=}$ área ocupada por uma classe de vazão; Pixels study area = área total de estudo

$$
P R_{\text {Pesos }}=\frac{\left(\text { Pixels overlap } / \text { Pixel }_{\text {evid }}\right)}{\left(\text { Pixels }_{\text {classe } \left._{\mathfrak{l}} / \text { Pixels }_{\text {study area }}\right)}\right.} \quad \text { Equação } 1
$$

Neste estudo, todos os temas evidenciais e os pontos de treinamento foram transformados ou reamostrados para imagens raster com células de $300 \mathrm{~m}$.

\section{Resultados}

Os resultados dos cálculos das probabilidades aparecem nas Figuras 4a,b,c,d,e. As associações entre domínios magnéticos e classes de vazão de poços são significativas e positivas. As chances de se encontrar poços com grandes vazões em terrenos magnéticos são três vezes ou mais do que em terrenos não magnéticos $(R P \geq 3)$. Por outro lado, quaisquer das classes de vazões possuem chances de não estarem associadas a domínios não magnéticos do que em outros domínios (associação negativa, $-4 \leq \mathrm{RP} \leq-1$ ). Terrenos de pouca declividade estão pouco associados às vazões. No entanto, maiores declividades estão negativamente associadas às vazões (RP $\leq-2)$, ou seja, as chances de não ocorrem maiores do que duas vezes. Quanto a litologia, a maioria não possui uma associação positiva com vazões. No entanto, o basalto microfanerítico Campo Erê é preditivo para a maior classe de vazão (RP $=1,72$ ) e o basalto com textura intergranular Campos Novos é preditivo para a menor classe de vazão $(\mathrm{RP}=$ 2.62). Com relação aos lineamentos estruturais, as maiores vazões (classes 5, 4 e 3) associam-se fortemente às orientações NW-SE e NNW-SSE. Orientações N-S e E-W relacionam-se às classes de menor vazão. Sobre os gradientes magnéticos orientados para N-S e NE-SW existem alta probabilidade de maiores 
vazões (classes 5, 4 e 3), enquanto que a classe 1 está relacionada às orientações $\mathrm{E}-\mathrm{W}$, semelhante aos lineamentos estruturais. Em relação aos poços-teste, quatro poços possuem áreas de influência sobre a Formação Campo Erê, todos de classes 4 e 5 de altas vazões.

\section{Discussão e Conclusões}

Por ser um projeto em andamento, os resultados das probabilidades de associações são consistentes quanto a amostragem de pontos de treinamento (100 poços), todavia eles não devem ser considerados no sentido quantitativo mas de modo qualitativo. O prosseguimento da pesquisa com a inserção de outras técnicas de modelagem e fontes de dados (imagens orbitais, informações de campo e de poços...) poderão aferir os resultados aqui apresentados. Por enquanto, os resultados revelam que regiões mais favoráveis para a locação de poços estão sobre basalto microfanerítico, com anomalia magnética positiva. Esta favorabilidade é aumentada com existência, em subsuperfície, de gradientes magnéticos orientados para o quadrante norte e nordeste e, em superfície, de lineamentos estruturais direcionados para o quadrante norte e nor-noroeste.

\section{Agradecimentos}

Os autores agradecem à CPRM e a SANEPAR pela cessão de informações e apoio institucional à pesquisa e ao estudante de geologia da UFPR Gabriel Fontanella.

\section{Referências}

ENCAL S.A., 1989. Projeto aeromagnetométrico Borda Oeste da Bacia do Paraná-Bloco Leste - Relatório Final de Aquisição e Processamento de Dados (Airborne magnetics project Western of Paraná Basin-East block Final Report on acquisition and processing). 3 vol. e anexos.

Cordell L. \& Graunch, V. J. S., 1985. Mapping basement magnetization zones from aeromagnetic data in the San Juan Basin, New Mexico. In: Hinze WJ (ed). The Utility of Regional Gravity and Magnetic Anomalies Maps. Society of Exploration Geophysicists, p.181-197.

Ferreira, F.J. F.; Souza, J. de, Bongiolo, A. de B. e S.; Castro, L. G. de; Romeiro, M. A. T., (neste simpósio). Realce do gradiente horizontal total de anomalias magnéticas usando a inclinação do sinal analítico. Parte I - Aplicação a dados sintéticos.

Lee, G. K; McCafferty, A. E.; Alminas, H. V.; Bankey, V.; Frishman, D.; Knepper, D. H. Jr.; Kulik, D. M.; Marsh, S. P.; Phillips, J. D.; Pitkin, J. A.; Smith, S. M.; Stoeser, D. B. Tysdal, R. G.; Van gosen, B. S., 2001. Montana geoenvironmental explorer: U.S. Geological Survey Digital Data Release DDS-65, Denver, 2001. Volume original de 1999, USGS Digital Data Series-XX.
Miller, H. G.; \& Singh V., 1994. Potential field tilt - a new concept for location of potential field sources. Journal of Applied Geophysics, 32(2-3): 213-217.

Nabighian, M. N., 1972. The analytic signal of twodimensional magnetic bodies with polygonal crosssection: Its properties and use for automated anomaly interpretation. Geophysics, 37(3): 507-517.

Nabighian, M. N., 1974. Additional comments on the analytic signal of two-dimensional magnetic bodies with polygonal cross-section. Geophysics, 39(1): 85-92.

Rosa Filho, E.F.; Bittencourt, A.V.L.; Salamuni, R. Contribuição ao estudo das águas subterrâneas nos basaltos no Estado do Paraná. Boletim Paranaense de Geociências, n.37, p. 22-41, 1987.

Rosa Filho, E. F.; Hindi, E. C. 2006. Diagnóstico das águas subterrâneas no Estado do Paraná: quantidade e Qualidade. Relatório técnico.

Rabus B.; Eineder, M.; Roth, A.; Bamler R., 2003. The Shuttle Radar Topography Mission - a new class of digital elevation models acquired by spaceborne radar. ISPRS Journal of Photogrammetry \& Remote Sensing. Elsevier Ed., n. 57, p. 241-262.

Roest, W. R.; Verhoef, J.; Pilkington, M., 1992. Magnetic interpretation using the 3-D analytic signal, Geophysics, $57(1): 116-125$.

Schobbenhaus, C., Gonçalves, J.H., Santos, J.O.S., Abram, M.B., Leão Neto, R., Matos, G.M.M. e Vidotti, R.M. (eds.)., 2003. Carta Geológica do Brasil ao Milionésimo (Geologic Chart of Brazil) Programa Levantamentos Geológicos Básicos do Brasil, CPRM, Brasília. 35 CD-ROMs. 

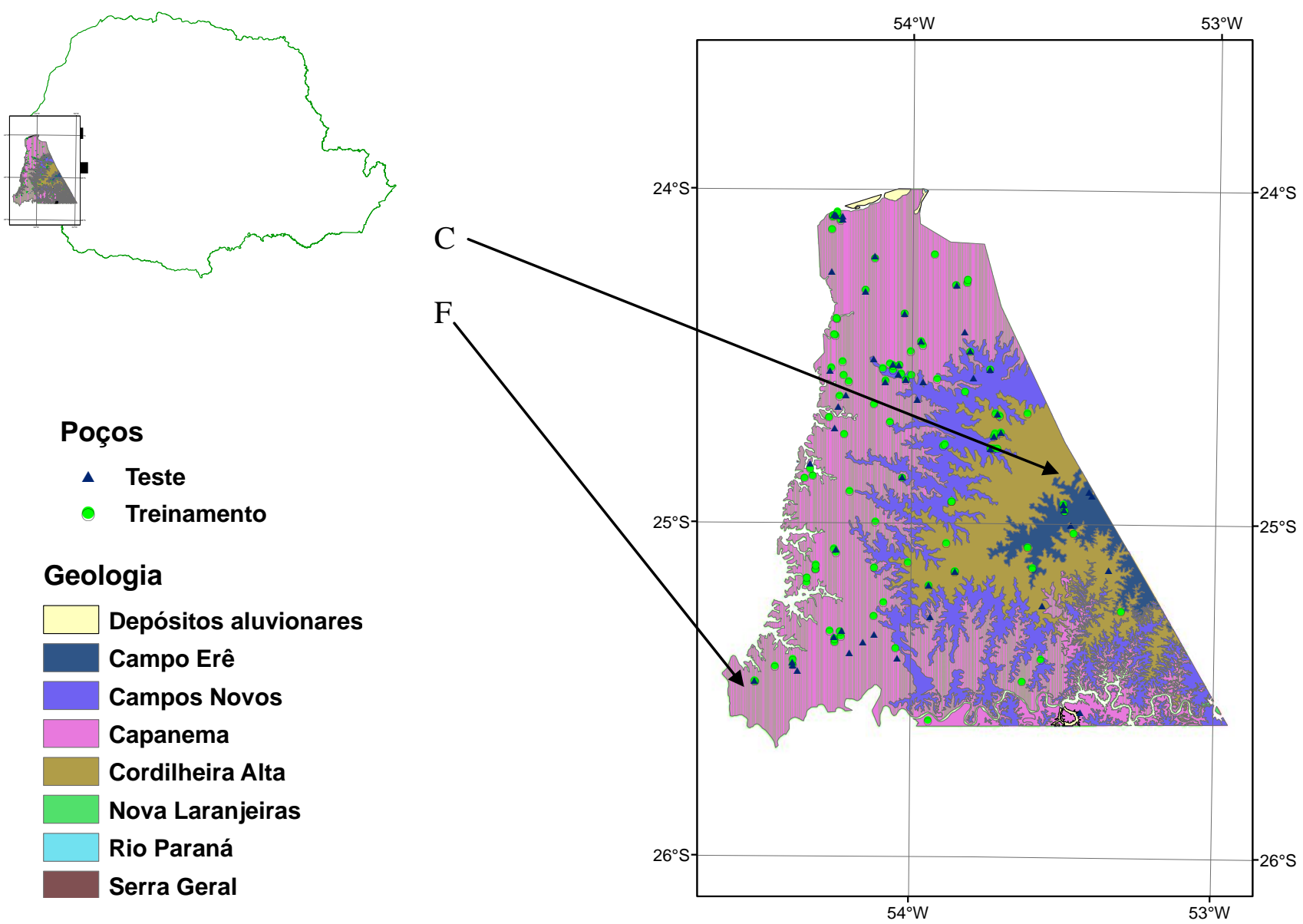

Figura 1 - Localização da área de estudo. Cidades: Cascavel (C); Foz do Iguaçu (F).

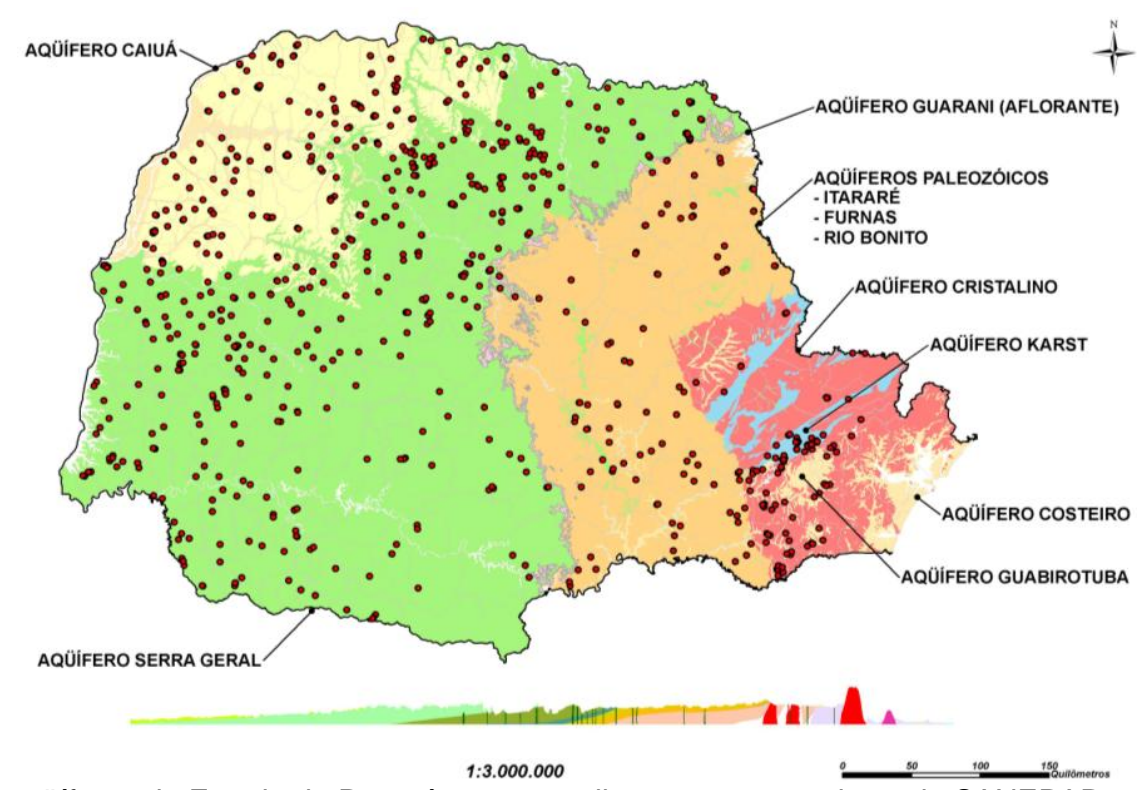

- Teste

- Treinamento

\section{Geologia}

Depósitos aluvionares

Campo Erê

Campos Novos

Capanema

Cordilheira Alta

Nova Laranjeiras

Rio Paraná

Serra Geral

Figura 2 - Principais aqüíferos do Estado do Paraná, em vermelho, poços operados pela SANEPAR. 


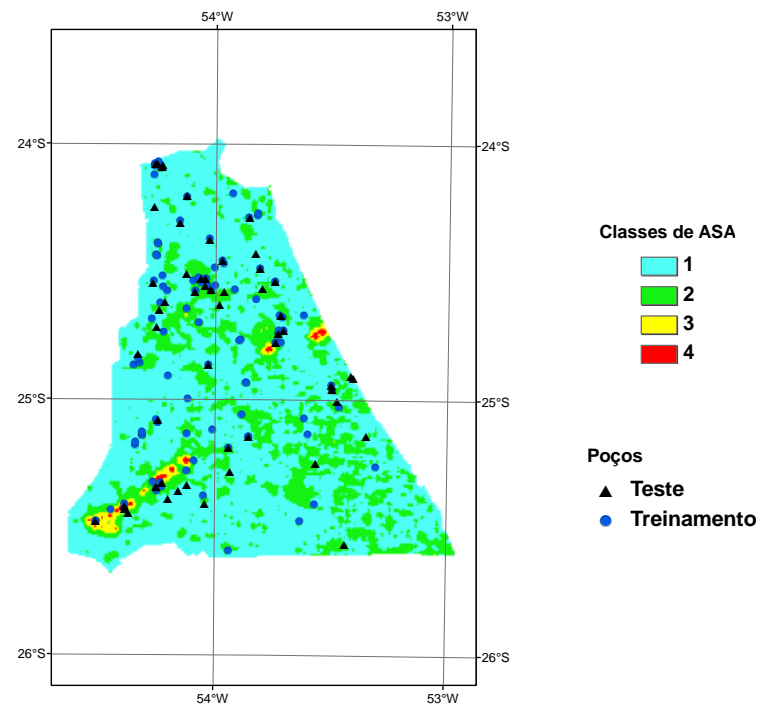

Figura 3a e 3b-Domínios magnéticos representados por classes da amplitude do sinal analítico calculado continuação ascendente a $500 \mathrm{~m}$ do campo magnético. 


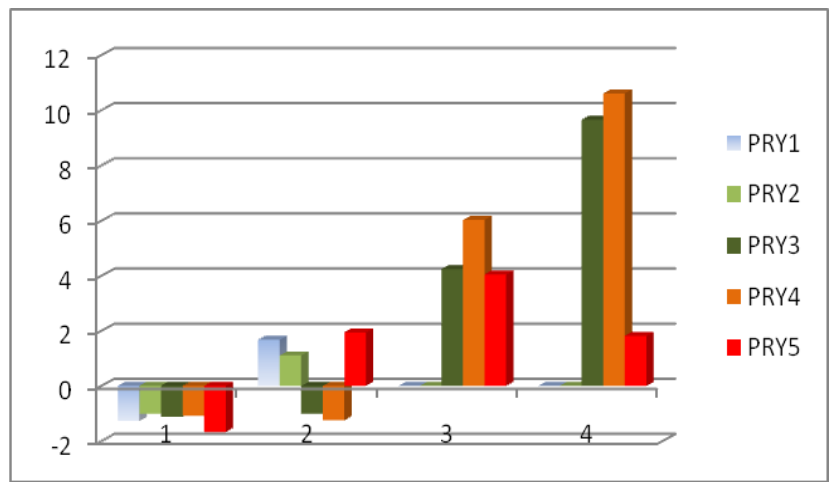

a) ASA

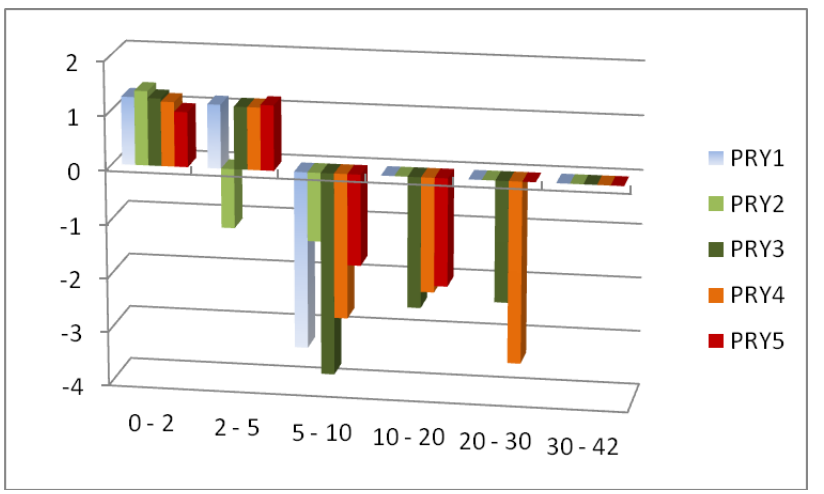

c) Slope

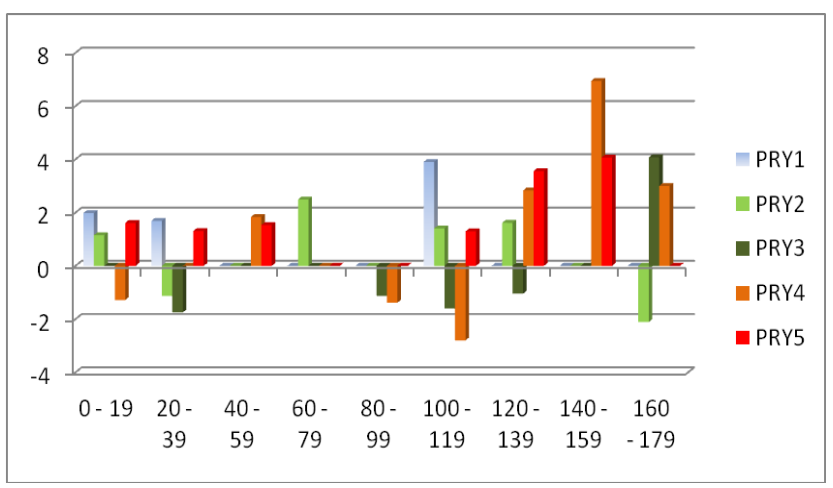

e) Lineamentos estruturais

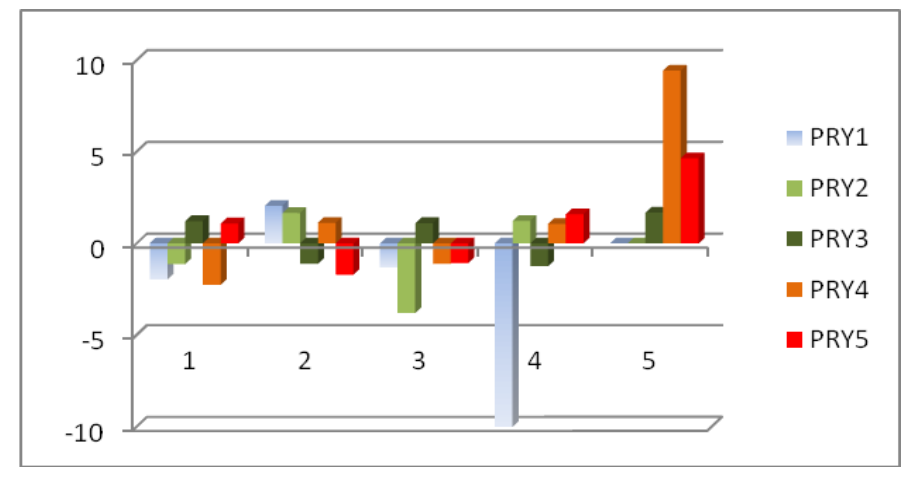

b) MAg

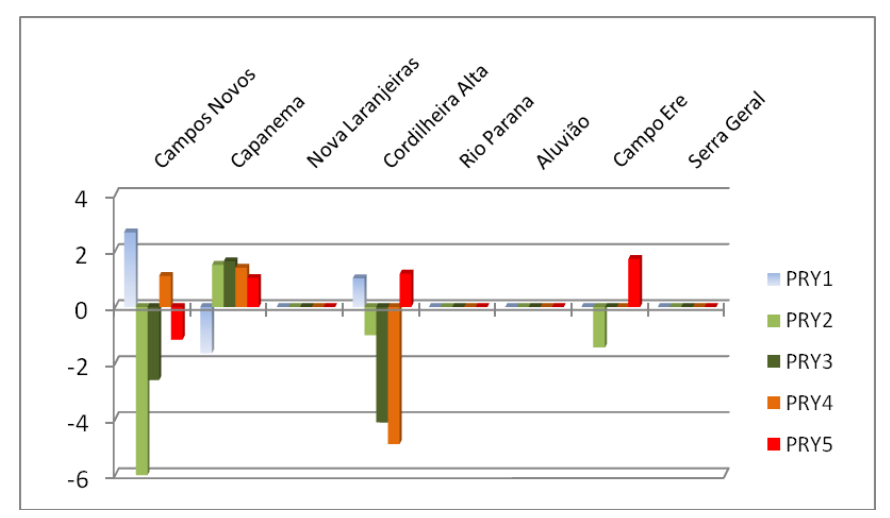

d) Litologia do Grupo Serra Grande e Depósitos Aluvionares

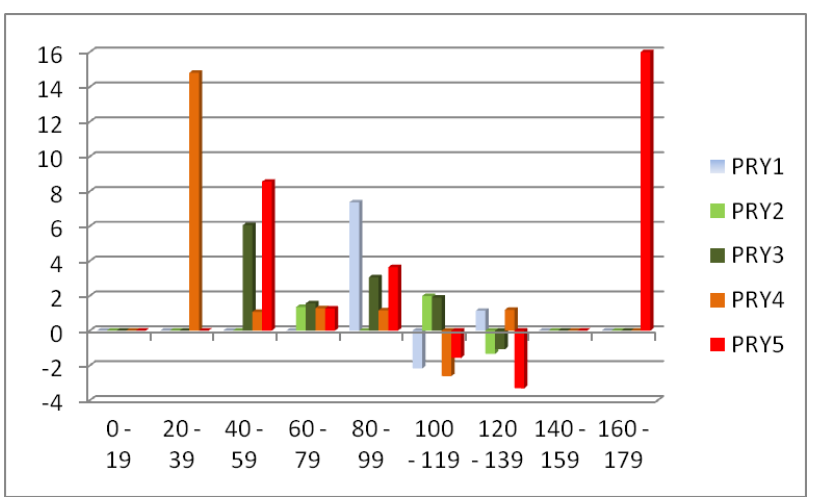

f) Gradientes magnéticos orientados

Figura 4 - Representação das probabilidades de associações entre temas evidenciais e classes de vazões de poços. Classes de temas evidenciais: ASA-amplitude do sinal analítico; CAM-campo magnético anômalo; declividade do terreno; litologia,orientação de lineamentos estruturais; orientação de gradientes magnéticos. 